\title{
Current Status and Future Perspectives of Immunotherapy in Middle-Income Countries: A Single-Center Early Experience
}

\author{
Imad Bou Akl ${ }^{\mathrm{a}}$, Juliett Berro ${ }^{\mathrm{b}}$, Arafat Tfayli ${ }^{\mathrm{b}}$, Ali Shamseddine ${ }^{\mathrm{b}}$, Deborah Mukherji ${ }^{\mathrm{b}}$, \\ Sally Temraz ${ }^{b}$, Jean El Cheikh ${ }^{b}$, Ibrahim A. Alameh ${ }^{b}$, Hazem I. Assi ${ }^{b, c}$
}

\begin{abstract}
Background: Immunotherapy agents offer novel treatment options in advanced cancers. However, their use is limited in developing countries lacking unifying guidelines and can be followed by a financial burden. In this study, we aimed to provide an overview regarding the use of immunotherapy and the overall response to treatment in patients with metastatic disease in relation to cost-effectiveness.
\end{abstract}

Methods: This was a retrospective study involving adult metastatic cancer patients, treated with programmed cell death-1 (PD-1) inhibitors at American University of Beirut Medical Center (AUBMC), a tertiary cancer center in Lebanon. Study enrollment began on January 1, 2014 and ended on January 12, 2016. Baseline demographics, epidemiological and clinical data were collected from the patients' records.

Results: Our study consisted of 34 patients. Fifteen patients selffinanced the treatment. The patients were prescribed immunotherapy without programmed cell death-ligand 1 (PD-L1) testing as it was not part of the guidelines at the time. Twenty-two patients were treated with nivolumab and 12 patients with pembrolizumab. Thirteen patients showed partial response or stable disease, while 21 patients showed progression.

Conclusion: Improvement in terms of overall survival and progression-free survival has been undercut by the lack of availability of these drugs and their cost. Considering that a large percentage of patients do not respond to immunotherapy, there is a need to use guide-

Manuscript submitted March 12, 2020, accepted March 31, 2020

Published online August 10, 2020

aDepartment of Internal Medicine, Division of Pulmonary and Critical Care Medicine, American University of Beirut Medical Center, Beirut, Lebanon ${ }^{b}$ Department of Internal Medicine, Hematology and Oncology, Naef K. Basile Cancer Institute, American University of Beirut Medical Center, Beirut, Lebanon

${ }^{\mathrm{c} C o r r e s p o n d i n g ~ A u t h o r: ~ H a z e m ~ I . ~ A s s i, ~ D e p a r t m e n t ~ o f ~ I n t e r n a l ~ M e d i c i n e, ~ H e-~}$ matology and Oncology, Naef K. Basile Cancer Institute, American University of Beirut Medical Center, PO Box 11-0236, Riad El Solh 1107 2020, Beirut, Lebanon. Email: ha157@aub.edu.lb

doi: https://doi.org/10.14740/wjon1277 lines such as a preset PD-L1 level that ensure cost-effectiveness and prevent resource waste.

Keywords: Immuno-oncology; Immunotherapy; PD-1/PD-L1 inhibitors; Cost-effective; Middle-income countries

\section{Introduction}

In the past few years, scientists have recently discovered and implemented novel use of immunotherapy, an effective but more expensive approach than traditional therapies (i.e., chemotherapy and radiation therapy).

Cancer cells have the ability to mitigate the body's natural immune response by exploiting the self-tolerance mechanisms and up-regulating the immune-inhibitory receptors. Recently, the growing understanding of several immune checkpoints that offer tumors an immunosuppressive function has evoked a paradigm shift in cancer treatment [1].

Different approaches to targeting immune checkpoint pathways have shown promise; however, the emphasis was placed on developing monoclonal antibody inhibitors of receptors with immunosuppressive properties. Immune checkpoints are initiated and maintained primarily by various receptors, including programmed cell death-1 (PD-1), programmed cell death-ligand 1 (PD-L1) and programmed cell death-ligand 2 (PD-L2). The interaction between PD-1 and PD-L1 dampens the immune response by affecting T-cell proliferation and cytokine production. Interfering with this pathway has been shown to enhance the body's antitumor defenses. Amongst the specific target drugs are the human IgG4 anti-PD-1 monoclonal antibodies, nivolumab and pembrolizumab [1-4].

Since their introduction in October 2015, nivolumab and pembrolizumab have shown substantial response rates in several types of cancer. They opened a new frontier in the battle against cancer. The enthusiasm surrounding their introduction was tampered down by the high financial cost on the patient and the health care system along with a new type of toxicities related to autoimmune activation not seen with traditional lung cancer therapy, such as pneumonitis, hepatitis and endocrinopathies [5]. PD-L1 inhibitors steep price and novel care 
added burden that is even more prominent in developing countries [6].

In developing countries, providing cancer patients with adequate care is challenging already, and the discrepancy between high-income countries and low and middle-income countries (LMICs), in terms of cancer patients' access to care and its quality, is considerable. The availability of new immunotherapies and their distribution in developing countries remain limited, and when available, cost considerably limits its access [7]. Besides, in rural and less urbanized areas, the number of general oncologists is significantly below the population's requirements, let alone the number of immuno-oncology specialists. To deal with the new immune-related side effects, there is a need to train and employ specialists in immuno-oncology which will extend the cost of the use of these two immunotherapies far beyond their initial price tags [8].

The presence of healthcare coverage supported by the state in some middle-income countries, such as Lebanon, may alleviate part of the financial burden of the cancer patients but shift the cost to the governmental agencies and the healthcare system. In Lebanon, the use of expensive drugs targeted for a limited population, such as immunotherapy, constitutes $20 \%$ of the healthcare governmental spending, which is a huge burden on the healthcare system. This burden necessitates the implementation of national policies to regulate and optimize the use of these medications for the maximal benefit $[9,10]$. In both high-income and low-income countries, experts advise considering cost-effectiveness estimates when deciding on reimbursement. Some of the proposed policies ensure reimbursement only when immunotherapy is given for specified types of cancer that have shown optimal results $[11,12]$. This approach remains theoretical in many cases such as in Lebanon [13].

Determining predictive biomarkers of treatment response is of the utmost importance, as it can aid in selecting the patients most likely to benefit from treatment. In the pursuit of these biomarkers, extensive research was carried out, and several molecules stood out as prime candidates, but with no definitive answers offered yet [14-16]. PD-L1 expression was found to be the only biomarker to predict therapy response, despite complex interplay with PD-L2 in the tumor microenvironment [17]. Although PD-L1-positive tumors respond better to immunotherapy, some studies showed that even tumors with $1 \%$ staining of PD-L1 still showed a median overall survival (OS) of more than 8 months [16].

Our research study is the first that tackles the issue of costeffectiveness of immunotherapy in relation to OS and treatment complications in Lebanon and the Middle East. In this study, we also looked at the current practices, the overall response to therapy, as well as the rate of discontinuation of treatment.

\section{Patients and Methods}

This was a retrospective single-institution study involving all adult patients $(\geq 18$ years old) with metastatic cancer, treated with PD-1 inhibitors at American University of Beirut Medical Center (AUBMC), a tertiary cancer center in a middle-income country in the Middle East.
Ethical approval was obtained from the American University of Beirut Medical Center (AUBMC) Institutional Review Board (IRB) on February 3, 2016 (IM.HA.05).

After obtaining IRB approval, identification data for cancer patients treated with immunotherapy was collected from the medical registry. Our research team had access to the patient's data records, with the cooperation and supervision of the patients' attending physicians throughout data collection. Study enrollment began on January 1, 2014 and ended on January $12,2016$.

Baseline demographics and epidemiological data were collected from the patients' records, in addition to clinical data including the type and stage of cancer, metastatic status, pre-treatment, dosage and type of immunotherapy, number of treatment cycles, pathological data, and response to treatment. Finally, rates of adverse events, patient's availability for follow-up, as well as financial coverage of the patient were collected.

To calculate the time-to-event in OS, the Kaplan-Meier curves were plotted using the time period between the date of diagnosis till the date of last follow-up for censored patients, in case the patient did not have a time of death. All analysis were performed using IBM SPSS statistics v.25.

\section{Results}

\section{Patient characteristics}

Our study cohort consisted of 34 patients. The median age of patients was 61 , with a mean age of 58 . The patient population consisted of 25 males and nine females. The majority of the cases were lung cancer $(n=17)$ and melanoma $(n=7)$. The remaining were divided among Hodgkin's lymphoma $(n=4)$, bladder cancer $(n=3)$, soft tissue sarcoma, gastric cancer and renal cell carcinoma $(\mathrm{n}=1$ each). The patients' demographics are summarized in Table 1.

Patient coverage data were available for 33 patients. The National Social Security Fund (NSSF) covered 14 patients, four patients were covered by health insurance companies and third-parties including the army, and 15 patients self-financed the treatment.

\section{Malignancy status}

Each of the 34 patients underwent either a biopsy or fineneedle aspiration (FNA) prior to treatment with chemotherapy or immunotherapy. However, while BRAF, EGFR and ALK mutations were reported, none of the patients had PD-L1 expression level results. PD-L1 testing was introduced in 2015 and was uncommon until recently in Lebanon. The patients who were recruited and followed up were prescribed immunotherapy without PD-L1 testing. Lymphocyte infiltration was negative in five patients, and reports from other patients did not include the results.

Thirty-three patients received chemotherapy treatment before immunotherapy. The median number of treatment lines 
Table 1. Patient Characteristics

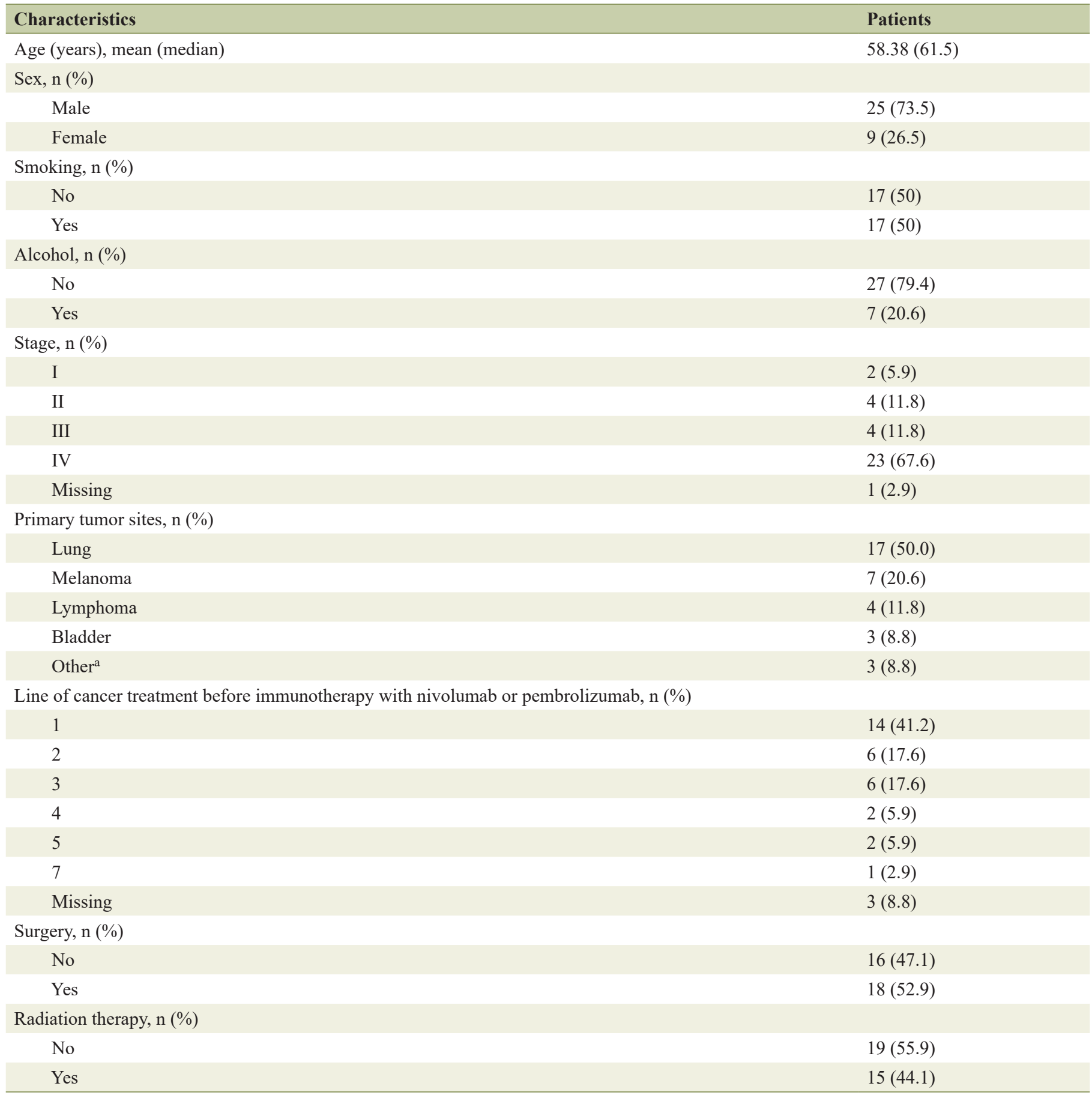

aSoft tissue sarcoma, gastric cancer and renal cell carcinoma.

received prior to immunotherapy was 2. Eighteen patients underwent surgery for their malignancies, while 15 patients received radiotherapy.

Twenty-two patients were treated with nivolumab and 12 patients with pembrolizumab. The mean number of immunotherapy cycles received by patients was 6 , with a median number of four cycles. Thirteen patients showed partial response or stable disease, while 21 patients had disease progression. Details of the patients' treatment characteristics with immunotherapy (nivolumab or pembrolizumab) are listed in Table 2 and Figure 1.

In terms of treatment complications, the majority of side effects were grade 1 and 2, with four patients developing grade 3 complications, including a patient developing pneumonitis. 
Table 2. Outcomes of Immunotherapy With Nivolumab or Pembrolizumab

\begin{tabular}{|ll}
\hline Characteristics & Patients \\
\hline $\begin{array}{l}\text { Immunotherapy, n (\%) } \\
\text { Nivolumab }\end{array}$ & $22(64.7)$ \\
Pembrolizumab & $12(35.3)$ \\
Line of immunotherapy, n (\%) & \\
1 & $2(5.9)$ \\
2 & $15(44.1)$ \\
3 & $6(17.6)$ \\
4 & $7(20.6)$ \\
5 & $1(2.9)$ \\
6 & $2(5.9)$ \\
8 & $1(2.9)$ \\
Complications, $\mathrm{n}(\%)$ & \\
No & $23(67.6)$ \\
Yes & $11(32.4)$ \\
\hline
\end{tabular}

In total, 11 patients developed adverse events. Treatment of side effects was mostly supportive, with steroids given to the patient with pneumonitis.

\section{Treatment status}

Discontinuation of treatment occurred in 14 patients, and treatment was interrupted in one patient's case. Ten patients received treatment after immunotherapy. Nineteen patients are still alive and receiving treatment either with immunotherapy or chemotherapy, 15 patients have died and three were lost to follow-up and excluded from the study. Patients were recruited in the period extending from January 1, 2014 to January 12, 2016. Patients were followed up until either death or discontinuation of treatment. Median follow-up time for patients since the date of diagnosis was 23 months. The median OS in all patients was 272.99 days (95.0\% confidence interval: 207.54 - 338.45) (Fig. 2a). The median OS by type of cancer were 198, 121 and 45 months for patients with lung, melanoma and other, respectively. None of the patients who had Hodgkin's lymphoma or bladder cancer passed away, therefore mean and median survival for these patients was not computed (Fig. 2b).

\section{Cost of treatment}

According to the Pharmacy Department at the AUBMC, the current price of a single injection of $40 \mathrm{mg}$ nivolumab is $1,927,000$ LL $(\$ 1,284)$, while an injection of $100 \mathrm{mg}$ nivolumab costs $4,624,000 \mathrm{LL}(\$ 3,089)$, and an injection of $100 \mathrm{mg}$ pembrolizumab is $8,219,000 \mathrm{LL}(\$ 5,479)$. According to the World Bank, $70 \%$ of the population in Lebanon generates an annual income of less than USD10,000, which was estimated based on the average salaries per sector, taking into consideration the level of experience and the job titles in Lebanon for 2016. Furthermore, the gross domestic product (GDP) per capita is USD8,523. In other words, one course of nivolumab can exceed the annual salary of a majority of the Lebanese population.

\section{Discussion}

This study addressed the use of immunotherapy, its cost, and

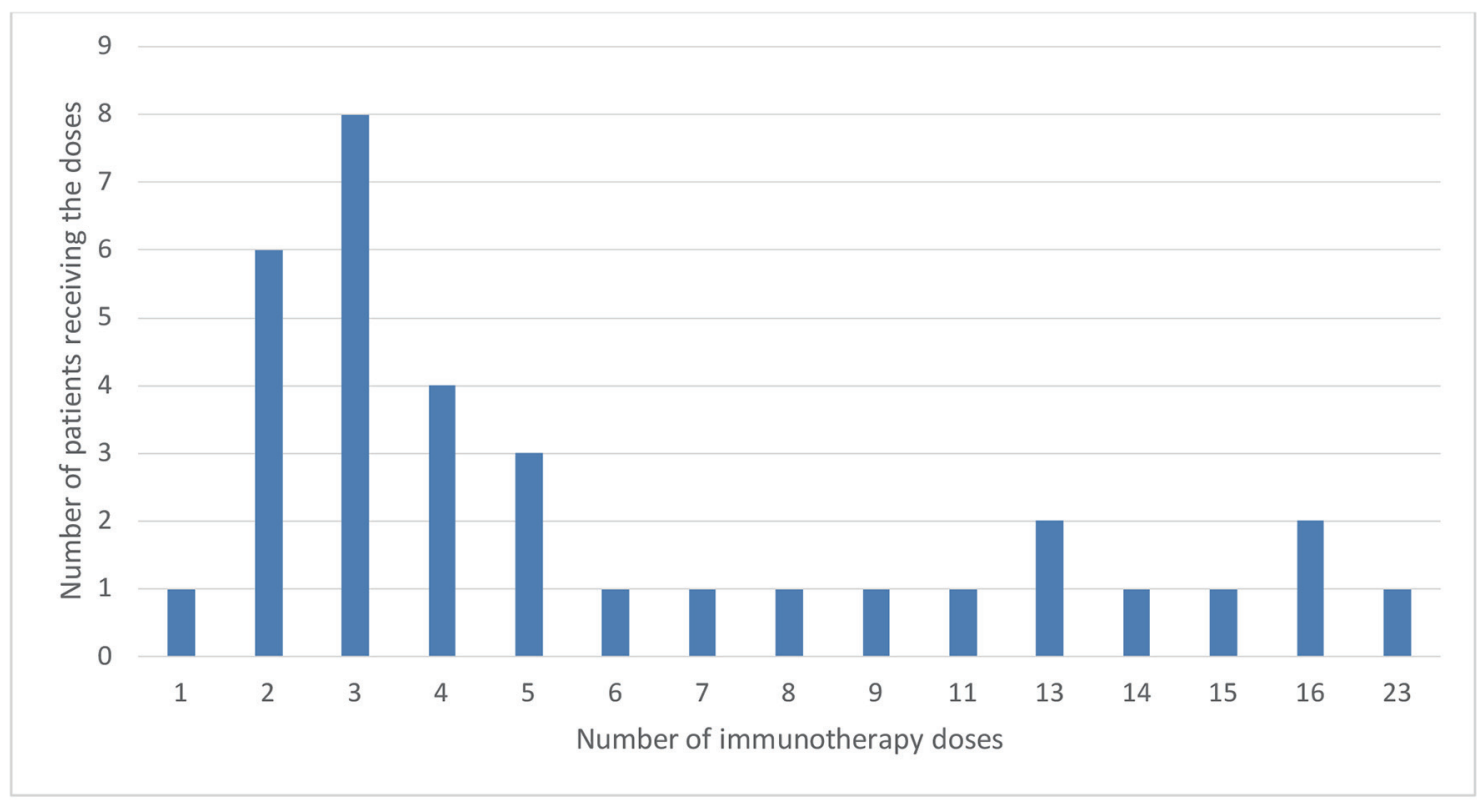

Figure 1. Number of immunotherapy doses. 


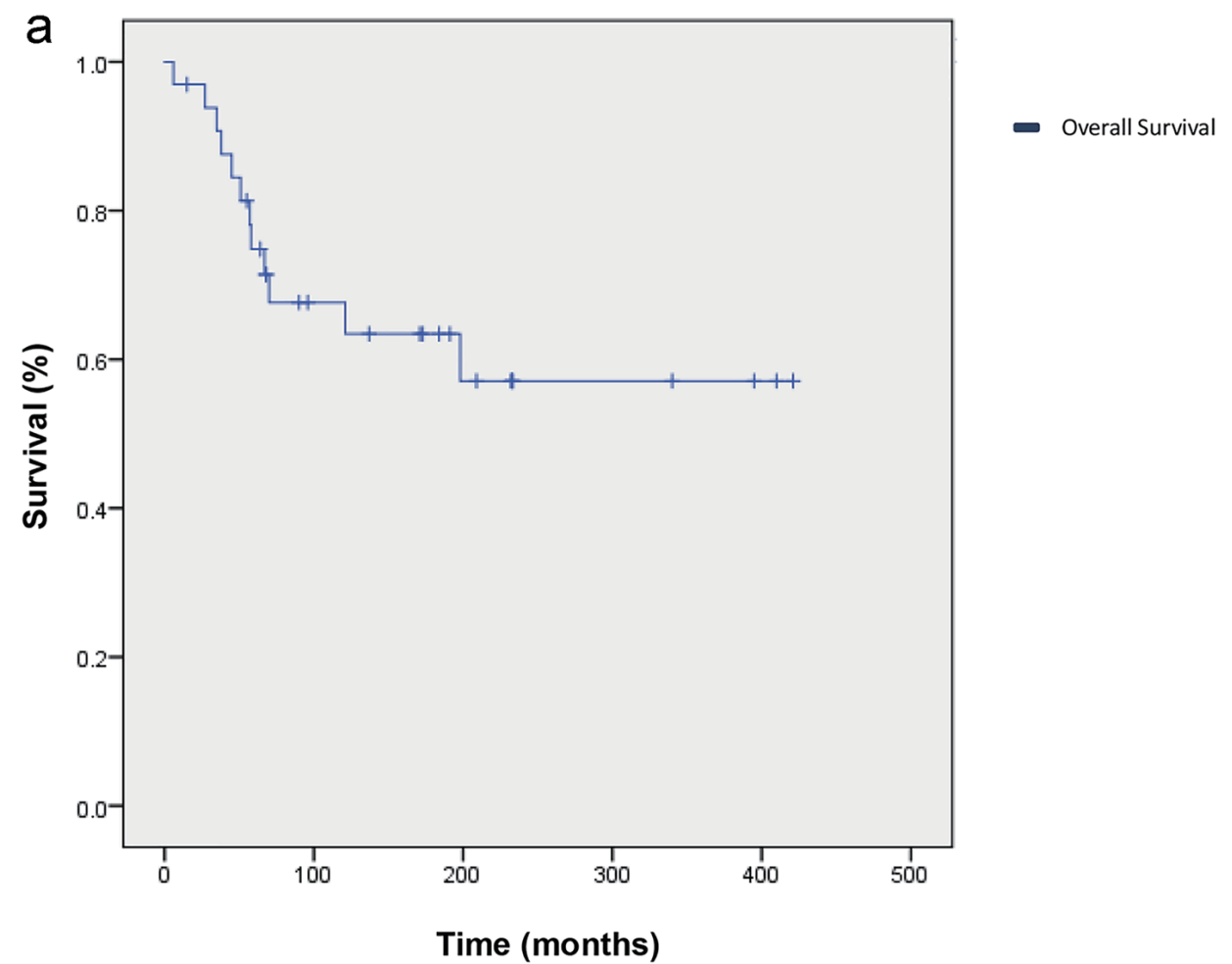

b

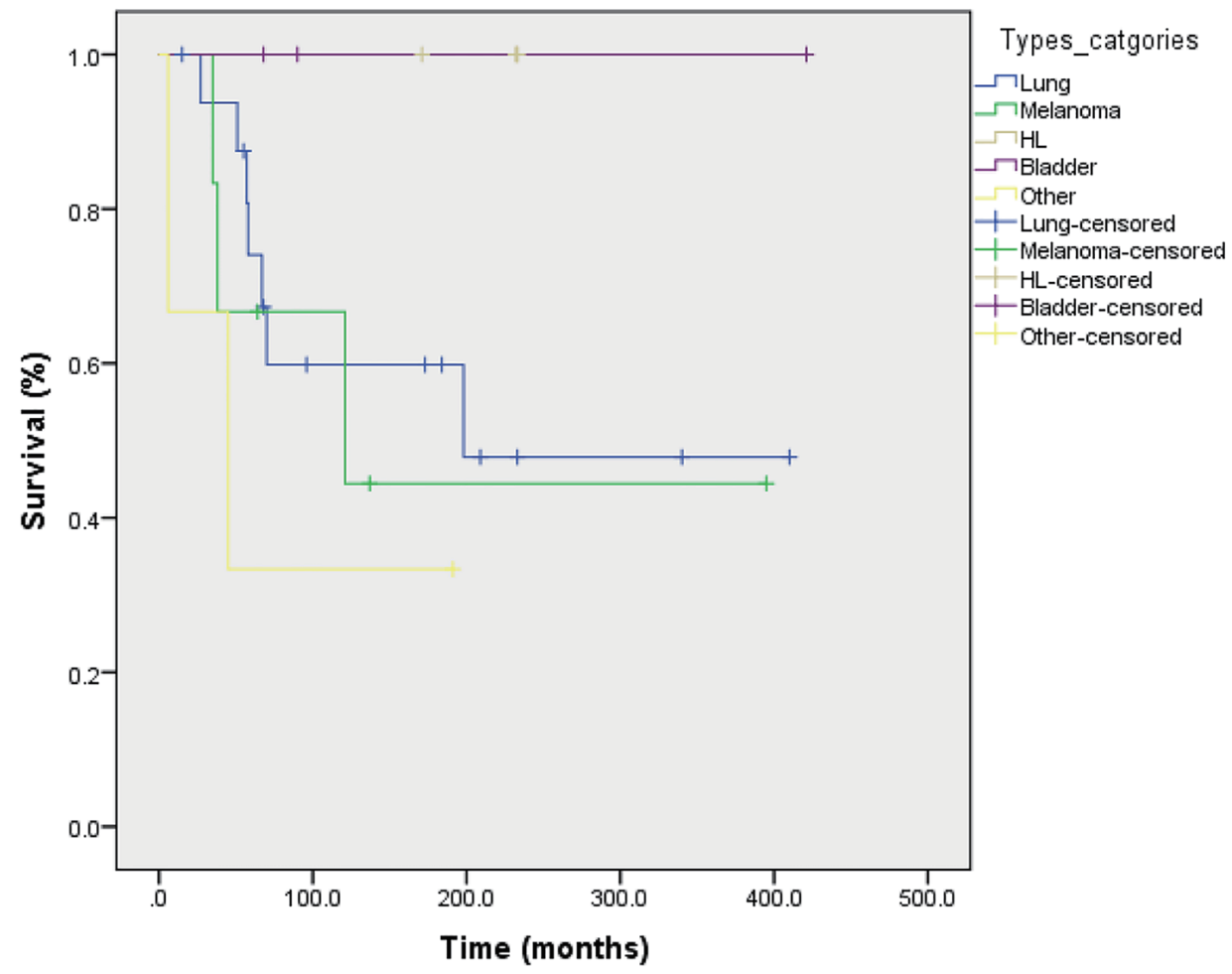

Figure 2. (a) Kaplan-Meier curve of overall survival (OS) for 33 cancer patients who received immunotherapy with either nivolumab or pembrolizumab between January 1, 2014 and January 12, 2016. The mean OS in all patients was 272.99 days $(95.0 \%$ confidence interval: 207.54 - 338.45). (b) Kaplan-Meier curve of OD according to the type of cancer. The median OSs by type were 198, 121 and 45 months for patients with lung, melanoma and other, respectively. None of the patients who had Hodgkin's lymphoma or bladder cancer passed away, therefore mean and median survival for these patients were not computed. 
the OS and complications associated with this therapy in Lebanon. We notice the lack of PD-L1 testing and lymphocyte infiltration reports. In 2015, PD-L1 testing was introduced in Lebanon but was not widely available until 2016. In our sample, no patient underwent PD-L1 testing. It could be explained by the lack of widespread laboratories that test for these markers at the time of our study. Recently published and presented clinical trials continue to confirm that outcomes are better for patients whose tumors have increasing levels of PD-L1 [17].

The lack of PD-L1 and lymphocyte infiltration testing may partially explain the rate of response to therapy. In our cohort, $21(61.8 \%)$ patients had disease progression, with treatment being discontinued for $14(41.2 \%)$ patients. Only four $(11.8 \%)$ patients show partial response to therapy, while one $(2.9 \%)$ patient showed complete response to treatment. While chemotherapy centers are widespread across our region, centers that offer immunotherapy are scarce. Hence, with more than half of the cohort having disease progression despite immunotherapy, it might not be practical for patients to go through the trouble in the first place. Moreover, the study revealed a mean OS of 272.99 days ( 9 months). The mean OS for nivolumab was 288.32 days (9.5 months), and that of pembrolizumab was 241.7 days ( 8 months), which is comparable to other studies in pretreated patients with advanced malignancies. Nivolumab showed slightly better survival than pembrolizumab.

Immunotherapy drugs brought along immune-related adverse events (irAEs) that required the attention of specialists and subspecialists [7]. These irAEs are not as frequent as standard chemotherapy side effects; however, they are more life-threatening $[7,18]$. In our cohort, there was no issue with following up with patients and treating irAEs. Patients were compliant with follow-up, except for three patients. The median follow-up time in our cohort was 23 months since the date of diagnosis. Only one patient had a severe irAEs manifesting as pneumonitis.

With an average cycle dose of $240 \mathrm{mg}$ for nivolumab and $100 \mathrm{mg}$ for pembrolizumab, a single cycle can cost $\$ 7,462$ for nivolumab and $\$ 10,958$ for pembrolizumab. The mean number of immunotherapy cycles given in our institution is 6 , running the cost of treatment to between $\$ 44,772$ and $\$ 65,748$. One patient received 13 cycles of nivolumab, raising the cost of treatment to $\$ 97,006$. Adding to these numbers, the travel cost, test prices, and the administrative costs, the price for immunotherapy treatment can be crippling. The annual expenses of nivolumab in Lebanon are comparable to the reported expenses of nivolumab in South Africa [19], and the costs per injection are similar to those reported in the United States $[9,10]$. It is also essential to notice that the average income in Lebanon, being less than USD10,000, is a fraction of the average annual income in the US $(\$ 59,039)$. The high cost of treatment is discouraging and raises concerns regarding the cost of future treatment. Even if healthcare systems provide patients with cancer treatment via third-party payers (government programs or private corporations), the rising costs of novel cancer treatments that show statistically significant results in clinical trials will ultimately push the absolute cost to a level that is unaffordable by the community [8]. It is even more real in middleincome countries, such as Lebanon. According to the report of the United Nations Development Program (UNDP), Lebanon is a high human development index (HDI) country. Despite the classification, income inequality persists, and the healthcare system still lacks appropriate governmental support. The current state in Lebanon spotlights the situation of many low and very-low HDI countries.

While it is true that immunotherapy has shown promising results in terms of survival benefits, one cannot ignore its financial burden on patients themselves. In Lebanon, some of the patients, as mentioned earlier, have to cover the cost of therapy. Even patients with third-party payers, such as the ministry of health coverage, will have out-of-pocket expenditures, depleting patients' savings, and ultimately limiting their access to treatment when the costs become intolerable. It has been shown that financial burdens can cause patients to stop treatment even when the benefit is evident or may drive others into declaring bankruptcy [9].

Improved patient selection is predicted to alleviate the economic burden of PD-1 inhibitors. This approach is limited by the dearth of knowledge regarding patient selection criteria. PD-L1 tumor levels showed the most promise in predicting response; however, the results have been inconsistent, with wide ranges of response to immunotherapy [14]. Considering the high costs of PD-1 inhibitors and the improved response rate in patients with elevated PD-L1 level, third-party payers might consider limiting drug use to a certain PD-L1 level threshold. Some countries, such as Serbia, have done that. Immunotherapy is only given to patients in whom exact indications apply [16].

Immunotherapy has been established as a staple in the treatment paradigm of many types of cancer, with the list of approved indications expanding at an accelerated rate. Physicians often feel compelled to go off-label, in an attempt to benefit patients whom they think have no other choice. This practice, however, imposes psychological burdens on patients who expect positive results, contrary to current scientific data. With the rapid expansion of available immunotherapies, such as avelumab, which was approved in 2017, and their widening indications, it is pivotal to streamline therapy. The choice of first, second and third-line treatments should be based on clinical data and established guidelines. This concept is highlighted in developing countries where resources are limited, and therefore should be employed in a manner that optimizes cost-benefit for patients.

In our center, patients receiving immunotherapy were not tested for PD-L1 expression. Drug discontinuation was common in our population, a multifactorial phenomenon that includes cost, practicality and logistics. More importantly, substantial clinical benefit was only observed in a minority of patients. We know from recent studies on immunotherapy for cancer such as anti-PD-1/PD-L1 and anti-cytotoxic T-lymphocyte associated protein 4 (anti-CTLA-4), that the immune system can control many cancers across various histologies and can produce durable response not seen with other treatment modalities. Studies have also shown that less than $25 \%$ of patients treated with immunotherapy will respond [20,21]. These facts highlight the importance of patient selection to optimize response and minimize cost.

The limitations of this study include its retrospective nature and relatively small sample size. A prospective study should be performed to better evaluate the cost-effectiveness of immunotherapy in Lebanon, including a follow-up for a 
longer duration of time.

In conclusion, lack of availability of these drugs, as well as their high cost has hindered improvement in terms of OS and progression-free survival in the region. Besides, a large percentage of patients who undergo immunotherapy does not respond to treatment. Therefore, the need to use guidelines employing preset PD-L1 level is essential. These guidelines are crucial to allocate scarce resources to patients that would benefit most properly. More prospective studies are urgently needed to assess the effect and side effects of these novel therapies in the Lebanese and Middle Eastern populations to improve these local guidelines.

\section{Acknowledgments}

We thank the nursing staff for providing excellent care for our patients and the physicians in the Hematology and Oncology Division at the American University of Beirut Medical Center and collaborating physicians.

\section{Financial Disclosure}

There was not any financial support for this research.

\section{Conflict of Interest}

The authors have no conflict of interest to declare.

\section{Informed Consent}

Waiver of informed consent was obtained from the IRB.

\section{Author Contributions}

Imad Bou Akl: conceptualization, supervision and writing original draft; Juliett Berro: investigation, visualization and data analysis; Arafat Tfayli: writing original draft; Ali Shamseddine: writing original draft; Deborah Mukherji: writing original draft; Sally Temraz: writing original draft; Jean El Sheikh: writing original draft; Ibrahim Alameh: project administration, writing, review and editing; Hazem I. Assi: conceptualization, supervision, writing, review and editing.

\section{Data Availability}

Any inquiries regarding supporting data availability of this study should be directed to the corresponding author.

\section{References}

1. Dolan DE, Gupta S. PD-1 pathway inhibitors: changing the landscape of cancer immunotherapy. Cancer Control. 2014;21(3):231-237.

2. Keir ME, Butte MJ, Freeman GJ, Sharpe AH. PD-1 and its ligands in tolerance and immunity. Annu Rev Immunol. 2008;26:677-704.

3. Gajewski TF, Louahed J, Brichard VG. Gene signature in melanoma associated with clinical activity: a potential clue to unlock cancer immunotherapy. Cancer J. 2010;16(4):399-403.

4. Hiraoka N. Tumor-infiltrating lymphocytes and hepatocellular carcinoma: molecular biology. Int J Clin Oncol. 2010;15(6):544-551.

5. Kim ES. Avelumab: first global approval. Drugs. 2017;77(8):929-937.

6. Goldstein DA, Stemmer SM, Gordon N. The cost and value of cancer drugs - are new innovations outpacing our ability to pay? Isr J Health Policy Res. 2016;5:40.

7. Brigden M, Imbulgoda M. Delivering immuno-oncology therapies in the community oncology setting: Introduction of anti-PD-1 therapy into two community oncology programs. Oncology Exchange. 2016;15:10-14.

8. Siddiqui M, Rajkumar SV. The high cost of cancer drugs and what we can do about it. Mayo Clin Proc. 2012;87(10):935-943.

9. Smith KE. Projecting income and assets: what might the future hold for the next generation of medicare beneficiaries? 2012.

10. Himmelstein DU, Thorne D, Warren E, Woolhandler S. Medical bankruptcy in the United States, 2007: results of a national study. Am J Med. 2009;122(8):741-746.

11. Bach PB. New math on drug cost-effectiveness. N Engl J Med. 2015;373(19):1797-1799.

12. Cazap E, Magrath I, Kingham TP, Elzawawy A. Structural barriers to diagnosis and treatment of cancer in lowand middle-income countries: the urgent need for scaling up. J Clin Oncol. 2016;34(1):14-19.

13. Elias F, Bou-Orm IR, Adib SM, Gebran S, Gebran A, Ammar W. Cost of oncology drugs in the middle-eastern country of lebanon: an update (2014-2016). J Glob Oncol. 2018;4:1-7.

14. Choudhury N, Nakamura Y. Importance of immunopharmacogenomics in cancer treatment: Patient selection and monitoring for immune checkpoint antibodies. Cancer Sci. 2016;107(2):107-115.

15. Hoos A, Eggermont AM, Janetzki S, Hodi FS, Ibrahim R, Anderson A, Humphrey R, et al. Improved endpoints for cancer immunotherapy trials. J Natl Cancer Inst. 2010;102(18):1388-1397.

16. Garon EB, Rizvi NA, Hui R, Leighl N, Balmanoukian AS, Eder JP, Patnaik A, et al. Pembrolizumab for the treatment of non-small-cell lung cancer. N Engl J Med. 2015;372(21):2018-2028.

17. Taube JM, Klein A, Brahmer JR, Xu H, Pan X, Kim JH, Chen L, et al. Association of PD-1, PD-1 ligands, and other features of the tumor immune microenvironment with response to anti-PD-1 therapy. Clin Cancer Res. 2014;20(19):5064-5074.

18. Brigden M, Lam W, Spadafora S, El-Maraghi R, Whitlock P, Champion P. Update on community oncology 
practice in Canada: A view from the trenches. Oncology Exchange. 2015;14(1):12-16.

19. Herbst M. Fact Sheet on Immunotherapy (Nivolumab) now available in South Africa. Cancer Assosciation of South Africa. 2017.
20. Zugazagoitia J, Guedes C, Ponce S, Ferrer I, MolinaPinelo S, Paz-Ares L. Current challenges in cancer treatment. Clin Ther. 2016;38(7):1551-1566.

21. Ventola CL. Cancer immunotherapy, Part 3: challenges and future trends. P T. 2017;42(8):514-521. 